\title{
A Stochastic Model of Tumorigenesis with Defects of Spindle Geometry and Chromosome Segregation Errors
}

\author{
Bala Srinivasan ${ }^{1}$, Udayabaskaran Swaminathan ${ }^{2} \&$ Zahir Hussain Buhari ${ }^{3}$ \\ ${ }^{1}$ Department of Statistics, Madras Christian College (Autonomous), Tambaram, \\ Chennai 600059, Tamil Nadu, India. \\ ${ }^{2}$ Department of Mathematics, Vel Tech Dr. RR \& Dr. SR Technical University, Avadi, Chennai 600062, India. \\ ${ }^{3}$ Department of General Studies, Yanbu Industrial College, P.O. Box 30436, Yanbu Al Sinaiyah, Yanbu, \\ Kingdom of Saudi Arabia.
}

\begin{abstract}
In this paper, we analyses a stochastic model of tumorigenesis which takes into account the transient defects of mitotic spindle geometry and chromosome segregation errors in cell division process. The model is essentially a Markov branching process evolving in a random environment. Using the model, we predict that the two defects may specifically contribute to the tumorigenesis initiation and progression.
\end{abstract}

\section{Introduction}

Pease and Tirnauer [5] have reported that mitosis not only segregates the chromosomes but also decides cell fate and tissue architecture (spindle geometry). The spindle geometry plays an important role in determining whether cell division is symmetric (producing identical daughter cells) or asymmetric (resulting in different daughter cell contents, placements or fates). They have observed that daughter cells orient their spindles by rotating them parallel or perpendicular to the cell and in turn the spindle orientation controls the placement of daughter cells within a tissue, influencing tissue morphology. Tumor suppressor gene (TSG) has link between spindle orientation disorder and cancer.

Recently, Silkworth and Cimini [6] have considered the transient defects of mitotic spindle geometry and chromosome segregation errors in cell population growth. They discussed how these defects may specifically contribute to the carcinogenesis initiation and progression. This paper has motivated us to formulate a stochastic model of tumorigenesis which takes into account the above mentioned defects. Our model is based on the model of Kimmel and Stivers [4].Kimmel and Stivers [4] have considered a population model of a cell-division process in which the population consists of countably infinite types of individuals and the types are denoted by $\mathrm{j}=0,1,2 \ldots$ The individuals of the population evolve according to the following rules:

a) A particle of type $\mathrm{j} \geq 1$ divides into two newborns

(i) both belonging to type $\mathrm{j}+1$ with probability $\alpha_{1}$;

(ii) both belonging to type $\mathrm{j}-1$ with probability $\alpha_{2}$;

(iii) both belonging to type $\mathrm{j}$ with probability $\alpha_{3}=1-\alpha_{1}-\alpha_{2}$.

b) An individual of type $\mathrm{j}=0$ produces two newborns of type 0 only.

c) The process is initiated at time $\mathrm{t}=0$ by a single particle of given type $i$.

The above formulation describes the process of evolution of reversible drug resistance in cancer cells. Their model explains the unstable drug resistance caused by extra chromosomal gene amplification and it takes into account both the stochastic changes in the number of gene copies from one generation to another and the stochastic variability in cell lifetimes. They have obtained the expression for the expected number of cells with a given number of gene copies in terms of modified Bessel functions. They have fitted the model to experimental data and obtained estimates of the probabilities of gene amplification and de-amplification. Their paper justifies that purely stochastic mechanisms may explain the dynamics of reversible drug resistance of cancer cells. Gusev et al. [3] have proposed a stochastic model that describes the evolution of chromosome number in a population of dividing cells as a result of chromosome segregation errors (CSE). Their model is described by a branching random walk in a K- dimensional nonnegative integer space. They have examined the effects of CSE on survival of cells and clones as well as on the dynamics of population growth and chromosome number distributions. Based upon their results, they have suggested that the long-term dynamics of cell population growth depends upon the rate of segregation errors. Swierniak et al. [7] have considered the models of emergence of resistance of cancer cells to chemotherapy and used the theory of feedback systems to study the asymptotic properties of the process. Andersson and Hughes [2] have analysed the gene duplication-amplification (GDA) processes and stated that whenever cellular growth is restricted, escape from these growth restrictions often occurs by GDA events that resolve the selective problem. They have also observed that GDA may facilitate subsequent genetic change by allowing a population to grow with a time delay and lead to increase in size, thereby increasing the probability for 
subsequent adaptive mutations to occur in the amplified genes or in unrelated genes. The above studies indicate that the most suitable model to study the dynamics of cell population growth is the branching random walk subject to the occurrences of events such as GDA and CSE. Further these studies have also observed that growth restriction in the cell population may lead to the mutations to occur in the amplified genes. The notion of time delay in branching is very intrinsic in biological populations. However, no branching random walk model of cell division process with time delay is available in the literature for the study of the dynamics of a cell population in which the individuals perform GDA and CSE with time delay. To fill this gap, we propose and analyse, in the present paper, a Markov branching random walk model with time delay which describes the role of disorder in spindle orientation in cancer development and progression. For this model, we obtain the mean functions explicitly.

\section{The plan of the paper is as follows:}

In Section 2, we describe the present model. Section 3 provides the system of integral equations for the probability generating functions for the underlying stochastic processes of the population. The mean values of the levels of the populations are found in section 4.

\section{Description of the Model}

We consider an extension of the model of Kimmel and Stivers [4] by assuming that the population evolves with age-dependent branching. To be specific, we assume the following rules for branching:

1) The life-spans of all particles are independent and identically distributed exponential random variables with mean $1 / \lambda$

2) A particle can leave off springs only at the end of its life-time.

3) A particle of type $\mathrm{j} \geq 0$ leaves no descendants when its life time is less than or equal to a fixed positive constant T.

4) A particle of type $\mathrm{j} \geq 1$ divides into two newborns

(i) both belonging to type $\mathrm{j}+1$ with probability $\alpha_{1} \neq 0$;

(ii) both belonging to type $\mathrm{j}$ - 1 with probability $\alpha_{2} \neq 0$;

(iii) both belonging to type $\mathrm{j}$ with probability $\alpha_{3}=1-\alpha_{1}-\alpha_{2}$.

5) An individual of type $\mathrm{j}=0$ produces two newborns of type 0 only.

6) The process is initiated at time $\mathrm{t}=0$ by a single particle of given type $i>0$ :

\section{Our objective is}

To analyses the probability distribution of the infinite vector $\mathrm{X}(\mathrm{t})=\left(\mathrm{X}_{0}(\mathrm{t}) ; \mathrm{X}_{1}(\mathrm{t}) ; \ldots\right)$, where $\mathrm{X}_{j}(\mathrm{t})$ is the number of particles of type $j$ at time $t$.

\section{The Integral Equations for The P. G. F.'S of X(t)}

We observe that the infinite-dimensional vector process $\mathrm{X}(\mathrm{t})$ is a Markov process. To study the process, we define the conditional probability generating functions

$$
G_{i, j}(s, t)=\mathcal{E}\left[s^{X_{j}(t)} \mid X_{i}(0)=1\right],
$$

Where $\xi$ stands for the expectation operator. Using the Markov property and the Kroneckordelta $\delta_{i j}$; we obtain

Case (i) $\mathbf{t} \leq \mathbf{T}$ :

$$
G_{i, j}(s, t)=e^{-\lambda t} s^{\delta_{i j}}+\lambda \int_{0}^{t} e^{-\lambda u} \mathrm{~d} u, i, j \geq 0 ;
$$

Case (ii) $\mathbf{t}>\mathbf{T}$ :

$$
\begin{gathered}
G_{i, j}(s, t)=e^{-\lambda t} s^{\delta_{i j}}+\lambda \int_{0}^{T} e^{-\lambda u} \mathrm{~d} u \\
+\lambda \alpha_{1} \int_{T}^{t} e^{-\lambda u}\left\{G_{i+1, j}(s, t-u)\right\}^{2} \mathrm{~d} u \\
+\lambda \alpha_{2} \int_{T}^{t} e^{-\lambda u}\left\{G_{i-1, j}(s, t-u)\right\}^{2} \mathrm{~d} u \\
+\lambda \alpha_{3} \int_{T}^{t} e^{-\lambda u}\left\{G_{i, j}(s, t-u)\right\}^{2} \mathrm{~d} u, i \geq 1, \\
G_{0,0}(s, t)=e^{-\lambda t} s+\lambda \int_{0}^{T} e^{-\lambda u} \mathrm{~d} u+\lambda \int_{T}^{t} e^{-\lambda u}\left\{G_{0,0}(s, t-u)\right\}^{2} \mathrm{~d} u .
\end{gathered}
$$

Using the above equations, we find the mean of $\mathrm{X}_{j}(\mathrm{t})$ in the next section. 
We define

\section{The means $m_{i, j}(t)$}

$$
m_{i, j}(t)=\mathcal{E}\left[X_{j}(t) \mid X_{i}(0)=1\right] .
$$

Differentiating (3.2) with respect to $\mathrm{s}$, and putting $\mathrm{s}=1$, we get

$$
m_{i, j}(t)=e^{-\lambda t} \delta_{i j}, t \leq T, i, j=0,1,2, \cdots .
$$

Similarly, by differentiating (3.3) with respect to s; and putting s = 1; we get

$$
\begin{gathered}
m_{i, j}(t)=e^{-\lambda t} \delta_{i j}+2 \lambda \alpha_{1} \int_{T}^{t} e^{-\lambda u} m_{i+1, j}(t-u) \mathrm{d} u \\
+2 \lambda \alpha_{2} \int_{T}^{t} e^{-\lambda u} m_{i-1, j}(t-u) \mathrm{d} u \\
+2 \lambda \alpha_{3} \int_{T}^{t} e^{-\lambda u} m_{i, j}(t-u) \mathrm{d} u, t>T ; i \geq 1, j \geq 0 .
\end{gathered}
$$

By differentiating (3.4) with respect to $\mathrm{s}$, and putting $\mathrm{s}=1$, we get

$$
m_{0, j}(t)=e^{-\lambda t} \delta_{0 j}+2 \lambda \int_{T}^{t} e^{-\lambda u} m_{0, j}(t-u) \mathrm{d} u, t>T ; j \geq 0 .
$$

The two equations (4.2) and (4.3) give

$$
\begin{gathered}
m_{i, j}(t)=e^{-\lambda t} \delta_{i j}+2 \lambda \int_{0}^{t} e^{-\lambda u} H(u-T)\left[\alpha_{1} m_{i+1, j}(t-u)\right. \\
\left.+\alpha_{2} m_{i-1, j}(t-u)+\alpha_{3} m_{i, j}(t-u)\right] \mathrm{d} u, i \geq 1, j \geq 0 .
\end{gathered}
$$

Using Laplace transform, (4.5) yields

$$
\begin{gathered}
m_{i, j}^{*}(\theta)=\frac{\delta_{i j}}{\theta+\lambda}+\frac{2 \lambda e^{-(\theta+\lambda) T}}{\theta+\lambda}\left[\alpha_{1} m_{i+1, j}^{*}(\theta)+\alpha_{2} m_{i-1, j}^{*}(\theta)\right. \\
\left.+\alpha_{3} m_{i, j}^{*}(\theta)\right], i=1,2, \cdots ; j \geq 0 .
\end{gathered}
$$

From (4.2) and (4.4), we get

$$
m_{0, j}(t)=e^{-\lambda t} \delta_{0 j}+2 \lambda \int_{0}^{t} H(u-T) e^{-\lambda u} m_{0, j}(t-u) \mathrm{d} u, j \geq 0 .
$$

Using Laplace transform, (4.7) yields

From (4.8), we get

$$
m_{0, j}^{*}(\theta)=\frac{\delta_{0 j}}{\theta+\lambda}+\frac{2 \lambda e^{-(\theta+\lambda) T}}{\theta+\lambda} m_{0, j}^{*}(\theta), j \geq 0 .
$$

$$
m_{0, j}^{*}(\theta)=\frac{\delta_{0 j}}{\theta+\lambda-2 \lambda e^{-(\theta+\lambda) T}} .
$$

Inverting (4.9), we obtain

$$
m_{0, j}(t)=\delta_{0 j} e^{-\lambda t} \sum_{k=0}^{\infty} \frac{\{2 \lambda(t-k T)\}^{k}}{k !} H(t-k T) .
$$

To solve the infinite system of equations (4.6), we define

$$
\mathbf{M}_{j}^{*}(\xi, \theta)=\sum_{i=0}^{\infty} m_{i, j}^{*}(\theta) \xi^{i}, j \geq 0 .
$$

Then, by using (4.6), we get

$$
\begin{aligned}
\mathbf{M}_{j}^{*}(\xi, \theta) & =m_{0, j}^{*}(\theta)+\xi^{j}\left(\frac{1-\delta_{0 j}}{\theta+\lambda}\right)+\frac{2 \lambda e^{-(\theta+\lambda) T}}{\theta+\lambda} \frac{\alpha_{1}}{\xi}\left\{\mathbf{M}_{j}^{*}(\xi, \theta)-\xi m_{1, j}^{*}(\theta)-m_{0, j}^{*}(\theta)\right\} \\
& +\alpha_{2} \xi \frac{2 \lambda e^{-(\theta+\lambda) T}}{\theta+\lambda} \mathbf{M}_{j}^{*}(\xi, \theta)+\alpha_{3} \frac{2 \lambda e^{-(\theta+\lambda) T}}{\theta+\lambda}\left\{\mathbf{M}_{j}^{*}(\xi, \theta)-m_{0, j}^{*}(\theta)\right\} .
\end{aligned}
$$


Solving for $M^{*}{ }_{j}(\xi, \theta) ;(4.12)$ gives

$$
\begin{gathered}
\mathbf{M}_{j}^{*}(\xi, \theta)=\frac{-(\theta+\lambda) m_{0 j}^{*}(\theta) \xi-\xi^{j+1}\left(1-\delta_{0 j}\right)}{2 \lambda e^{-(\theta+\lambda) T} \alpha_{2} \xi^{2}-\left\{(\theta+\lambda)-2 \lambda e^{-(\theta+\lambda) T} \alpha_{3}\right\} \xi+2 \lambda e^{-(\theta+\lambda) T} \alpha_{1}} \\
+\frac{2 \lambda e^{-(\theta+\lambda) T}\left\{\alpha_{1} \xi m_{1 j}^{*}(\theta)+\alpha_{1} m_{0 j}^{*}(\theta)+\alpha_{3} \xi m_{0 j}^{*}(\theta)\right\}}{2 \lambda e^{-(\theta+\lambda) T} \alpha_{2} \xi^{2}-\left\{(\theta+\lambda)-2 \lambda e^{-(\theta+\lambda) T} \alpha_{3}\right\} \xi+2 \lambda e^{-(\theta+\lambda) T} \alpha_{1}} .
\end{gathered}
$$

The function $M^{*}{ }_{j}(\xi, \theta)$ is analytic in $\xi$ and its denominator vanishes at the points given by the quadratic equation

$$
2 \lambda e^{-(\theta+\lambda) T} \alpha_{2} \xi^{2}-\left\{(\theta+\lambda)-2 \lambda e^{-(\theta+\lambda) T} \alpha_{3}\right\} \xi+2 \lambda e^{-(\theta+\lambda) T} \alpha_{1}=0 .
$$

The roots of (4.14) are given by

$$
\xi_{1,2}=\frac{\kappa \mp \sqrt{\kappa^{2}-16 \lambda^{2} e^{-2(\theta+\lambda) T} \alpha_{1} \alpha_{2}}}{4 \lambda \alpha_{2} e^{-(\theta+\lambda) T}},
$$

Where $k=\theta+\lambda-2 \lambda e^{-(\theta+\lambda) T} \alpha_{3}$; We invoke the condition that $\lim \theta \rightarrow 0 m_{i j}^{*}(\theta)=0$ and it is satisfied only by the first root $\xi_{1}$.Then the numerator in (4.13) also vanishes at $\xi_{1}$. Consequently, we get

$$
m_{1 j}^{*}(\theta)=\frac{(\theta+\lambda) m_{0 j}^{*}(\theta) \xi_{1}+\xi_{1}^{j+1}\left(1-\delta_{0 j}\right)-2 \lambda e^{-(\theta+\lambda) T} m_{0 j}^{*}(\theta)\left\{\alpha_{1}+\alpha_{3} \xi_{1}\right\}}{2 \lambda e^{-(\theta+\lambda) T} \alpha_{1} \xi_{1}} .
$$

Putting $\mathrm{j}=0$ in (4.16), we get

$$
m_{10}^{*}(\theta)=\frac{m_{\mathrm{OO}}^{*}(\theta)\left[(\theta+\lambda) \xi_{1}-2 \lambda e^{-(\theta+\lambda) T}\left\{\alpha_{1}+\alpha_{3} \xi_{1}\right\}\right]}{2 \lambda e^{-(\theta+\lambda) T} \alpha_{1} \xi_{1}} .
$$

From (4.9), we get

$$
m_{\mathrm{O}, \mathrm{O}}^{*}(\theta)=\frac{1}{\theta+\lambda-2 \lambda e^{-(\theta+\lambda) T}} .
$$

Substituting (4.18) in (4.17), we get

$$
m_{10}^{*}(\theta)=\frac{(\theta+\lambda) \xi_{1}-2 \lambda e^{-(\theta+\lambda) T}\left\{\alpha_{1}+\alpha_{3} \xi_{1}\right\}}{2 \lambda \alpha_{1} \xi_{1} e^{-(\theta+\lambda) T}\left\{\theta+\lambda-2 \lambda e^{-(\theta+\lambda) T}\right\}} .
$$

For $j \neq 0,(4.9)$ gives $m_{0, j}^{*}(\theta)=0$ and consequently, (4.16) yields

$$
m_{1 j}^{*}(\theta)=\frac{\xi_{1}^{j}}{2 \lambda e^{-(\theta+\lambda) T} \alpha_{1}} .
$$

Substituting for $\xi_{1}(4.20)$ gives

$$
m_{1 j}^{*}(\theta)=\frac{1}{2 \lambda e^{-(\theta+\lambda) T} \alpha_{1}}\left[\frac{\kappa-\sqrt{\kappa^{2}-16 \lambda^{2} e^{-2(\theta+\lambda) T} \alpha_{1} \alpha_{2}}}{4 \lambda \alpha_{2} e^{-(\theta+\lambda) T}}\right]^{j} .
$$

In particular, when $\mathrm{j}=1 ;(4.21)$ gives 


$$
\begin{aligned}
& m_{11}^{*}(\theta)=\frac{\kappa}{8 \lambda^{2} e^{-2(\theta+\lambda) T} \alpha_{1} \alpha_{2}}\left[1-\sqrt{1-\frac{16 \lambda^{2} e^{-2(\theta+\lambda) T} \alpha_{1} \alpha_{2}}{\kappa^{2}}}\right] \\
= & \frac{\kappa}{8 \lambda^{2} e^{-2(\theta+\lambda) T} \alpha_{1} \alpha_{2}} \sum_{m=0}^{\infty} \frac{(2 m) !}{2^{2 m+1} m !(m+1) !}\left[\frac{16 \lambda^{2} e^{-2(\theta+\lambda) T} \alpha_{1} \alpha_{2}}{\kappa^{2}}\right]^{m+1} \\
= & \sum_{m=0}^{\infty} \frac{(2 m) !}{m !(m+1) !} \frac{2^{2 m} \lambda^{2 m} e^{-2 m(\theta+\lambda) T} \alpha_{1}^{m} \alpha_{2}^{m}}{\left\{\theta+\lambda-2 \lambda e^{-(\theta+\lambda) T} \alpha_{3}\right\}^{2 m+1}} \\
= & \sum_{m=0}^{\infty} \frac{(2 m) !}{m !(m+1) !} \frac{2^{2 m} \lambda^{2 m} e^{-2 m(\theta+\lambda) T} \alpha_{1}^{m} \alpha_{2}^{m}}{(\theta+\lambda)^{2 m+1}}\left\{1-\frac{2 \lambda e^{-(\theta+\lambda) T} \alpha_{3}}{\theta+\lambda}\right\}^{-(2 m+1)} \\
= & \sum_{m=0}^{\infty} \frac{(2 m) !}{m !(m+1) !} \frac{2^{2 m} \lambda^{2 m} e^{-2 m(\theta+\lambda) T} \alpha_{1}^{m} \alpha_{2}^{m}}{(\theta+\lambda)^{2 m+1}} \sum_{k=0}^{\infty}\left(\begin{array}{c}
2 m+k \\
k
\end{array}\right) \frac{2^{k} \lambda^{k} e^{-k(\theta+\lambda) T} \alpha_{3}^{k}}{(\theta+\lambda)^{k}} \\
= & \sum_{m=0}^{\infty} \sum_{k=0}^{\infty} \frac{(2 m) !}{m !(m+1) !}\left(\begin{array}{c}
2 m+k \\
k
\end{array}\right) \frac{2^{2 m+k} \lambda^{2 m+k} e^{-(2 m+k)(\theta+\lambda) T} \alpha_{1}^{m} \alpha_{2}^{m} \alpha_{3}^{k}}{(\theta+\lambda)^{2 m+k+1}} .
\end{aligned}
$$

Inverting (4.22), we get

$$
\begin{gathered}
m_{11}(t)=e^{-\lambda t} \sum_{m=0}^{\infty} \sum_{k=0}^{\infty} \frac{(2 m) !}{m !(m+1) !}\left(\begin{array}{c}
2 m+k \\
k
\end{array}\right) \\
\frac{H(t-(2 m+k) T)[2 \lambda\{t-(2 m+k) T\}]^{2 m+k} \alpha_{1}^{m} \alpha_{2}^{m} \alpha_{3}^{k}}{(2 m+k) !} \\
=e^{-\lambda t} \sum_{m=0}^{\infty} \sum_{k=0}^{\infty} \frac{H(t-(2 m+k) T)[2 \lambda\{t-(2 m+k) T\}]^{2 m+k} \alpha_{1}^{m} \alpha_{2}^{m} \alpha_{3}^{k}}{m !(m+1) ! k !} .
\end{gathered}
$$

When $\mathrm{T}=0$; equation (4.23) gives

$$
\begin{gathered}
m_{11}(t)=e^{-\lambda t} \sum_{m=0}^{\infty} \sum_{k=0}^{\infty} \frac{(2 \lambda t)^{2 m+k} \alpha_{1}^{m} \alpha_{2}^{m} \alpha_{3}^{k}}{m !(m+1) ! k !}=e^{-\lambda t} e^{2 \lambda \alpha_{3} t} \sum_{m=0}^{\infty} \frac{(2 \lambda t)^{2 m} \alpha_{1}^{m} \alpha_{2}^{m}}{m !(m+1) !} \\
=e^{-\lambda t} e^{2 \lambda\left\{1-\left(\alpha_{1}+\alpha_{2}\right)\right\} t} \sum_{m=0}^{\infty} \frac{\left(2 \lambda \alpha_{1} t\right)^{m}\left(2 \lambda \alpha_{2} t\right)^{m}}{m !(m+1) !}=e^{\lambda\left\{1-2\left(\alpha_{1}+\alpha_{2}\right)\right\} t} \sum_{m=0}^{\infty} \frac{\left(2 \lambda \alpha_{1} t\right)^{m}\left(2 \lambda \alpha_{2} t\right)^{m}}{m !(m+1) !} .
\end{gathered}
$$

It is known (see Abramowitz and Stegun [1]) that

$$
\sum_{m=0}^{\infty} \frac{z^{m}}{m !(m+1) !}=\frac{1}{\sqrt{z}} I_{1}(2 \sqrt{z}) \text {, }
$$

where $I_{1}(z)$ is the modified Bessel function. Taking $z=4 \lambda^{2} \alpha_{1} \alpha_{2} t^{2}$ in (4.25), we get

$$
\begin{aligned}
& I_{1}\left(2 \sqrt{4 \lambda^{2} \alpha_{1} \alpha_{2} t^{2}}\right)=\sqrt{4 \lambda^{2} \alpha_{1} \alpha_{2} t^{2}} \sum_{m=0}^{\infty} \frac{\left\{4 \lambda^{2} \alpha_{1} \alpha_{2} t^{2}\right\}^{m}}{m !(m+1) !} \\
& \sum_{m=0}^{\infty} \frac{\left\{2 \lambda \alpha_{1} t\right\}^{m}\left\{2 \lambda \alpha_{2} t\right\}^{m}}{m !(m+1) !}=\frac{1}{2 \lambda t \sqrt{\alpha_{1} \alpha_{2}}} I_{1}\left(4 \lambda t \sqrt{\alpha_{1} \alpha_{2}}\right) .
\end{aligned}
$$

Substituting (4.26) in (4.24), we deduce the result of Kimmel and Stivers [4]:

$$
m_{11}(t)=e^{\lambda\left\{1-2\left(\alpha_{1}+\alpha_{2}\right)\right\} t} \frac{1}{2 \lambda t \sqrt{\alpha_{1} \alpha_{2}}} I_{1}\left(4 \lambda t \sqrt{\alpha_{1} \alpha_{2}}\right) .
$$


For the general case $\mathrm{j} \geq 2$, we get

$$
\begin{gathered}
m_{1 j}^{*}(\theta)=\frac{1}{2^{2 j+1} \lambda^{j+1} e^{-(j+1)(\theta+\lambda) T} \alpha_{1} \alpha_{2}^{j}}\left[\kappa-\sqrt{\kappa^{2}-16 \lambda^{2} e^{-2(\theta+\lambda) T} \alpha_{1} \alpha_{2}}\right]^{j} \\
=\sum_{k=0}^{j} \sum_{r=0}^{\infty} \sum_{l=0}^{\infty}(-1)^{k+r+l}\left(\begin{array}{l}
j \\
k
\end{array}\right)\left(\begin{array}{l}
\frac{k}{2} \\
r
\end{array}\right)\left(\begin{array}{c}
j-2 r \\
l
\end{array}\right) \times \\
\frac{2^{4 r+l-2 j-1} \lambda^{2 r+l-j-1} e^{-(2 r+l-(j+1)\}(\theta+\lambda) T} \alpha_{1}^{r-1} \alpha_{2}^{r-j} \alpha_{3}^{l}}{(\theta+\lambda)^{2 r+l-j}} .
\end{gathered}
$$

Inverting (4.28), we get

$$
\begin{gathered}
m_{1 j}(t)=e^{-\lambda t} \sum_{k=0}^{j} \sum_{r=0}^{\infty} \sum_{l=0}^{\infty} \frac{(-1)^{k+r+l}}{(2 r+l-j-1) !}\left(\begin{array}{c}
j \\
k
\end{array}\right)\left(\begin{array}{c}
k / 2 \\
r
\end{array}\right)\left(\begin{array}{c}
j-2 r \\
l
\end{array}\right) \times 2^{4 r+l-2 j-1} \alpha_{1}^{r-1} \alpha_{2}^{r-j} \alpha_{3}^{l} \times \\
H[t-\{2 r+l-(j+1)\} T](\lambda[t-\{2 r+l-(j+1)\} T])^{2 r+l-j-1}, j \geq 2 .
\end{gathered}
$$

Now, (4.19) gives

$$
\begin{gathered}
m_{10}^{*}(\theta)=\frac{1}{\alpha_{1}} \sum_{k=0}^{\infty}\left[\frac{(2 \lambda)^{k-1} e^{-(k-1)(\theta+\lambda) T}}{(\theta+\lambda)^{k}}-\frac{\alpha_{3}(2 \lambda)^{k} e^{-k(\theta+\lambda) T}}{(\theta+\lambda)^{k+1}}\right] \\
-\sum_{k=0}^{\infty} \sum_{l=0}^{\infty} \sum_{r=0}^{\infty} \sum_{m=0}^{\infty}(-1)^{r}\left(\begin{array}{c}
\frac{l}{2} \\
r
\end{array}\right)\left(\begin{array}{c}
2 r+m \\
m
\end{array}\right) \frac{2^{k+4 r+m+2} \lambda^{k+2 r+m+1} e^{-(k+2 r+m+1)(\theta+\lambda) T} \alpha_{1}^{r} \alpha_{2}^{r+1} \alpha_{3}^{m}}{(\theta+\lambda)^{2 r+k+m+2}} .
\end{gathered}
$$

Inversion of (4.30) gives

$$
\begin{gathered}
m_{10}(t)=\frac{e^{-\lambda t}}{\alpha_{1}} \sum_{k=0}^{\infty}\left[\frac{(2 \lambda)^{k-1}\{t-(k-1) T\}^{k-1} H(t-(k-1) T)}{(k-1) !}\right. \\
\left.-\frac{\alpha_{3}(2 \lambda)^{k}(t-k T)^{k} H(t-k T)}{k !}\right]-e^{-\lambda t} \sum_{k=0}^{\infty} \sum_{l=0}^{\infty} \sum_{r=0}^{\infty} \sum_{m=0}^{\infty}(-1)^{r}\left(\begin{array}{c}
\frac{l}{2} \\
r
\end{array}\right)\left(\begin{array}{c}
2 r+m \\
m
\end{array}\right) \times \\
\frac{2^{2 r+1}(2 \lambda\{t-(k+2 r+m+1) T\})^{2 r+k+m+1} H(t-(k+2 r+m+1) T) \alpha_{1}^{r} \alpha_{2}^{r+1} \alpha_{3}^{m}}{(2 r+k+m+1) !} .
\end{gathered}
$$

When $\alpha_{1}=0$ and $\alpha_{2}=0$, we get $\alpha_{3}=1$ and consequently, (4.6) yields

$$
m_{i j}^{*}(\theta)=\frac{\delta_{i j}}{\theta+\lambda-2 \lambda e^{-(\theta+\lambda) T}}=\delta_{i j} \sum_{n=0}^{\infty} \frac{(2 \lambda)^{n} e^{-n(\theta+\lambda) T}}{(\theta+\lambda)^{n+1}} .
$$

Inverting (4.32), we obtain

$$
m_{i j}(t)=\delta_{i j} e^{-\lambda t} \sum_{n=0}^{\infty} \frac{(2 \lambda[t-n T])^{n} H(t-n T)}{n !} .
$$

\section{Bibliography}

[1]. Abramowitz, M. and Stegun, I. J., 1965. "Handbook of Mathematical Functions," Dover.

[2]. Andersson, D. I. and Hughes, D., 2009. "Gene Amplification and Adaptive Evolution inBacteria," Annual Review of Genetics, Vol. 43, pp. 167-195.

[3]. Gusev, Y., Kagansky, V. and Dooley, W. C., 2000. "A stochastic model of chromosomesegregation errors with reference to cancer cells," Mathematical and Computer Modelling,Vol. 32, pp. 97-111.

[4]. Kimmel, M. and Stivers, D. N., 1994. "Time-continuous branching walk models of unstablegene amplification," Bulletin of Mathematical Biology, Vol. 56, pp. 337-357.

[5]. Pease, J. C., and Tirnauer, J. S., 2011. "Mitotic spindle misorientation in cancer out ofalignment and into the fire," Journal of Cell Science, Vol. 124, pp. 1007-1016.

[6]. Silkworth, W. T., and Cimini, D., 2012. "Transient defects of mitotic spindle geometryand chromosome segregation errors," Cell Division, 7:19.

[7]. Swierniak, A., Polanski, A., Smieja, J. and Kimmel, M., 2003. "Modelling Growth ofDrug Resistant Cancer Populations as the System with Positive Feedback,” Mathematicaland Computer Modelling, Vol. 37, pp. 1245-1252. 